\title{
A potential significance of circ_0024169 down regulation in angiosarcoma tissue
}

\author{
Satoko Nakashima ${ }^{1}$, Masatoshi Jinnin ${ }^{1, *}$, Maho Ide', Ikko Kajihara ${ }^{1}$, Toshikatsu Igata ${ }^{1}$, \\ Miho Harada $^{1}$, Shinichi Masuguchi ${ }^{1}$, Satoshi Fukushima ${ }^{1}$, Mikio Masuzawa ${ }^{2}$, \\ Mamiko Masuzawa ${ }^{3}$, Yasuyuki Amoh ${ }^{3}$, Hironobu Ihn ${ }^{1}$ \\ ${ }^{1}$ Department of Dermatology and Plastic Surgery, Faculty of Life Sciences, Kumamoto University, Kumamoto, Japan; \\ ${ }^{2}$ Department of Molecular Diagnostics, School of Allied Health Sciences, Kitasato University, Sagamihara, Kanagawa, Japan, \\ ${ }^{3}$ Department of Dermatology, Kitasato University School of Medicine, Sagamihara, Kanagawa, Japan.
}

\begin{abstract}
Summary Circular RNAs (circRNAs) are recently characterized non-coding RNAs that have a closed continuous loop. CircRNAs might play important roles in the oncogenesis of several cancers. However, little is known about association between circRNAs and skin tumors. In this study, we tried to demonstrate the expression change of circ 0024169 in angiosarcoma, and to elucidate correlations between circ_0024169 expression in angiosarcoma tissues and clinical manifestation. RNA expression was evaluated by quantitative real-time PCR with TaqMan systems for circ_0024169 and linear isoform CUL5. Both relative circRNA levels (corrected for EEF1A1 levels) and circRNA levels/linear RNA expression ratio were evaluated. We found that both relative circ_0024169 levels and circ_0024169/CUL5 ratio was decreased in normal human dermal microvascular endothelial cells (HDMEC) and angiosarcoma cell line in vitro, compared to squamous cell carcinoma line. circ_0024169/ CUL5 ratio was significantly reduced in angiosarcoma and pyogenic granuloma than other tumors in vivo, which were more evident than decreased relative circ_0024169 levels. On the other hand, relative circ 0024169 levels showed mild inverse correlation with the follow-up periods (duration between the first hospital visit and the last hospital visit/the date of death) of angiosarcoma patients. Taken together, circ_0024169/CUL5 ratio are likely to be useful as a diagnostic biomarker for vascular tumors, whereas circ_0024169 levels may have more potential as a prognostic marker of angiosarcoma. The future studies of the function of circRNAs may lead to the clarification of detailed mechanism of oncogenesis of angiosarcoma.
\end{abstract}

Keywords: Angiosarcoma, circular RNA, epigenetics

\section{Introduction}

Angiosarcoma is a rare malignant vascular tumor originating from endothelial cells of blood/lymphatic vessels. The tumor usually occurs in the skin, especially the scalp and face of elderly people. Its aggressive

Released online in J-STAGE as advance publication May 14, 2019.

*Address correspondence to:

Dr. Masatoshi Jinnin, Department of Dermatology and Plastic Surgery, Faculty of Life Sciences, Kumamoto University, Honjo 1-1-1, Kumamoto 860-8556, Japan.

E-mail: jinjin1011@hotmail.com progression and resistance to standard chemotherapy result in frequent local recurrence and hematogenous metastasis to the lungs, pleura, or liver. The five-year survival rate of the patients is approximately 20-30\% (1-3), and angiosarcoma is therefore known as one of the tumors with very poor prognosis. However, the etiology of this tumor is still unknown.

Circular RNAs (circRNAs) are non-coding RNAs characterized by its circularized shape. circRNAs have a closed continuous loop formed by back-splice reactions that involve a covalent junction between the 3'-and 5'ends. This structure was firstly described in early 1990s and was initially considered as by-products of splicing errors $(4,5)$. However, recent comprehensive analysis of 
transcripts by high-throughput sequencing demonstrated that circRNAs are widely expressed in human cells (6). A detailed analysis of the circRNA sequences revealed that some circRNAs contain a large number of target sequences of specific microRNAs (miRNAs): miRNAs are short RNA molecules consisting of about 22 nucleotides, which exhibit gene regulatory functions by silencing target translation via interaction with its $3^{\prime}$ untranslated region (3' UTR). circRNAs are thought to bind specific miRNAs to its own sequence, and reduce intracellular active and free miRNA levels, thereby negatively regulate miRNA function. Such effects were firstly demonstrated using cultured cells (7), and then the phenotype shown by the forced overexpression of circRNA coincided with that shown by the suppression of corresponding miRNA expression in zebrafish. Accordingly, circRNAs are thought to serve as 'miRNA sponges' (6-8). Furthermore, another important feature of circRNAs is increased resistance to RNase due to the lack of ends attacked by exonucleases ( 6 ).

Recently, circRNA expression were shown to be tissue/cell-specific $(6,9)$, and are implicated in the tumorigenesis of several cancers (10-12). However, the role of circRNAs in skin tumors has not been examined. In this study, we tried to investigate the possibility that circRNAs may play roles in angiosarcoma, focusing on circ_0024169, which were reported to be dysregulated in oval cancer and colon cancer previously (13).

\section{Materials and Methods}

\subsection{Patient materials}

Skin specimens were obtained from five pyogenic granuloma, four malignant melanoma, three squamous cell carcinoma, seven normal subjects, and ten angiosarcoma. Seven control normal skin samples were from routinely discarded skin of healthy human subjects undergoing skin grafts. Institutional review board approval and written informed consent were obtained according to the Declaration of Helsinki before patients and healthy volunteers were entered into this study.

\subsection{Cell culture}

Adult human dermal microvascular endothelial cells (HDMEC) were obtained from Lonza (Walkersville, MD), and cultured according to the manufacturer's recommendations (14). The angiosarcoma cell line, ISO-HAS, was isolated from a tumor tissue specimen, and was grown as previously described (15). A human cutaneous squamous cell carcinoma (SCC) cell line, A431, was obtained from ATCC (Manassas, VA), and were cultured in DMEM (Lonza) with $10 \%$ fetal bovine serum (Hyclone, Logan, UT) and AntibioticAntimycotic (Invitrogen, Carlsbad, CA) in a $5 \% \mathrm{CO}_{2}$ incubator at $37^{\circ} \mathrm{C}(16)$.

\subsection{RNA isolation}

Total RNA isolation from cultured cells with RNeasy Mini Kit (Qiagen) and from paraffin-embedded sections with RNeasy FFPE kit (Qiagen, Valencia, CA) were performed according to the manufacturer's instructions, as described previously (13). The quality of RNA was evaluated using Agilent 2100 Bioanalyzer (Agilent Technologies, Santa Clara, CA).

\subsection{RNase R digestion, $c D N A$ synthesis, and quantitative real-time $P C R$}

Total RNA samples were pooled, digested by DNase I (Epicentre, Illumina, San Diego, CA), and treated with RNase R (Epicentre) twice to remove linear RNAs and enrich circular RNAs in QIAzol Lysis Reagent (Qiagen) (13).

First-strand cDNA was synthesized using PrimeScript RT reagent Kit (Takara) from the total RNA and RNase R-treated RNA. PCR was performed on Thermal Cycler DiceTM Real Time System (TP800; Takara, Shiga, Japan). Quantitative real-time PCR with TaqMan systems was performed with primers and templates mixed with Premix Ex Taq (Probe qPCR, Takara). Primer and probes for hsa_circ_0024169 were from Takara. Primers for CUL5 and EEF1A1 were from Life Technologies, Thermo Fisher Scientific (Waltham, MA). DNA was amplified for 50 cycles of denaturation for 5 seconds at $95^{\circ} \mathrm{C}$ and annealing for 30 seconds at $60^{\circ} \mathrm{C}$.

\subsection{Statistical Analysis}

Statistical analyses were carried out with Kruskal-Wallis test for the analysis of more than three groups, and Mann-Whitney tests were carried out for the comparison of medians between two groups. Correlations were evaluated by Pearson's correlation coefficient. $P$-values $<$ 0.05 were considered significant.

\section{Results}

\section{1. circ_0024169 expression in angiosarcoma cell lines in vitro}

We first examined the expression levels of circ_0024169 in angiosarcoma cell line ISO-HAS and HDMEC. SCC line A431 was also used for the disease control. TaqMan Gene Expression Assays were designed to distinguish between circ_0024169 and the corresponding linear isoform CUL5. Quantitative real-time PCR with TaqMan systems for the specific circular and linear isoform RNAs and for the housekeeping gene EEF1A1 was performed according to the previous report (13). Both relative circRNA levels corrected by housekeeping gene levels and circRNA levels/linear RNA expression ratio were evaluated. 
As a result, relative circ_0024169 levels corrected by EEF1A1 levels were lower in endothelial cells (HDMEC and ISO-HAS) than in A431 in vitro, especially in HDMEC (Figure 1A). Furthermore, similar tendency was found in the circ_0024169/CUL5 ratio (Figure 1B).

\section{2. circ_0024169 expression in angiosarcoma tissues in vivo}

Then, the relative circ_0024169 levels and circ_0024169/
CUL5 ratio in the tissue sections of normal skin $(n=$ $7)$, angiosarcoma ( $n=12)$, pyogenic granuloma $(n=$ $5)$, malignant melanoma $(n=4)$, and $\operatorname{SCC}(n=3)$ were determined with real-time PCR. There was no difference in the relative circ 0024169 levels between normal skin and angiosarcoma skin, although the levels tended to be increased in melanoma and SCC compared with normal skin (Figure 2A).

On the other hand, the circ_0024169/CUL5 ratio was highest in normal tissues than tumors. The values were
A

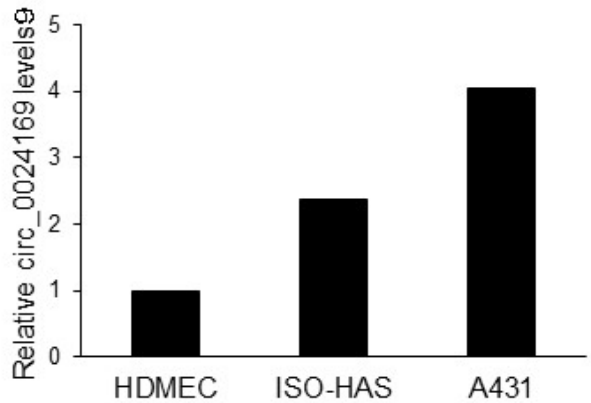

B

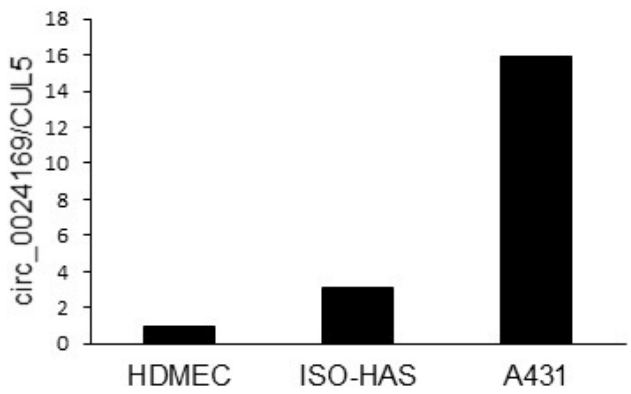

Figure 1. Expression of circ_0024169 in cultured HDMEC and ISO-HAS in vitro. The results of quantitative real-time PCR analyses to determine (A) the relative level of circ 0024169 (normalized to EEF1A1 levels) and (B) the expression ratio of circ 0024169 to its corresponding linear transcript (circ 0024169/CUL5) in cultured human dermal microvascular endothelial cells (HDMEC) and angiosarcoma cell line (ISO-HAS) were shown. The data in cultured SCC cell line (A431) was also shown as the disease control. The levels in HDMEC were set at 1.

A

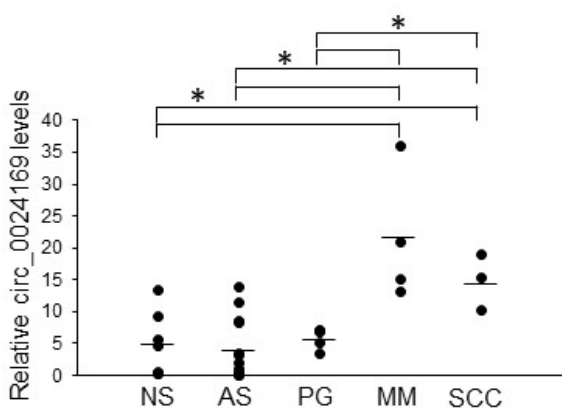

C

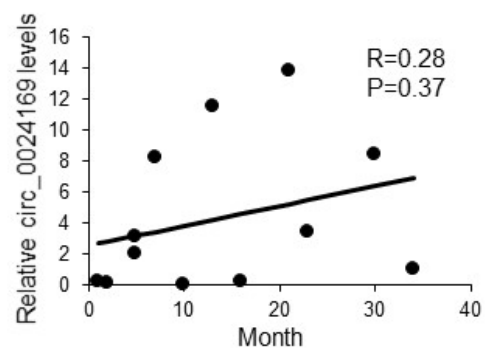

B

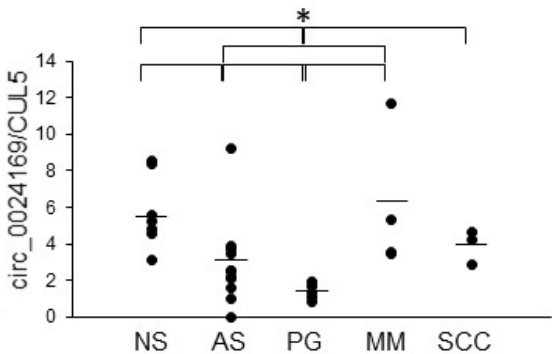

D

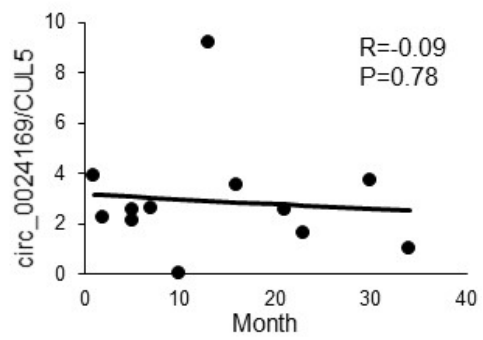

Figure 2. Expression of circ_0024169 in vascular tumors in vitro. Relative circ_0024169 levels (A) and the circ_0024169/ CUL5 ratio (B) in pyogenic granuloma ( $\mathrm{PG}, n=5)$, malignant melanoma (MM, $n=4)$, squamous cell carcinoma $(\mathrm{SCC}, n=3)$, normal skin (NS, $n=7$ ), and angiosarcoma (AS, $n=10$ ) were shown. (C, D) Association between circ 0024169 expression and clinical features in angiosarcoma. (C) Correlation of relative circ 0024169 levels (normalized to EEF1A 1 levels) with follow-up periods in patients with angiosarcoma was shown. (D) Correlation of circ 0024169/CUL5 ratio with follow-up periods was also presented. The minimum value in AS was set at 1 . Bars show means. ${ }^{*} p<\overline{0} .05$. 
A

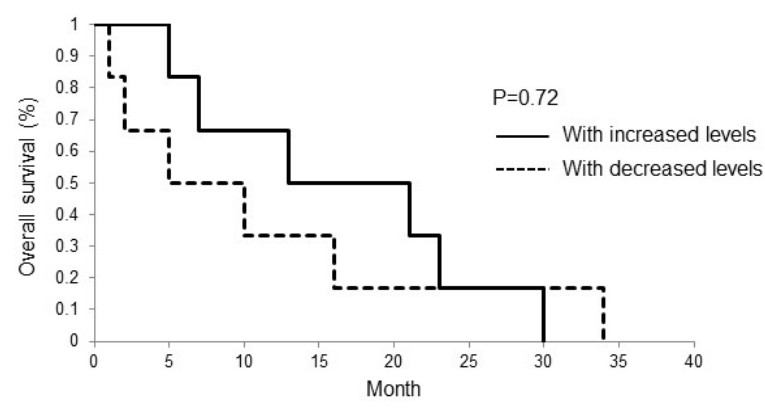

B

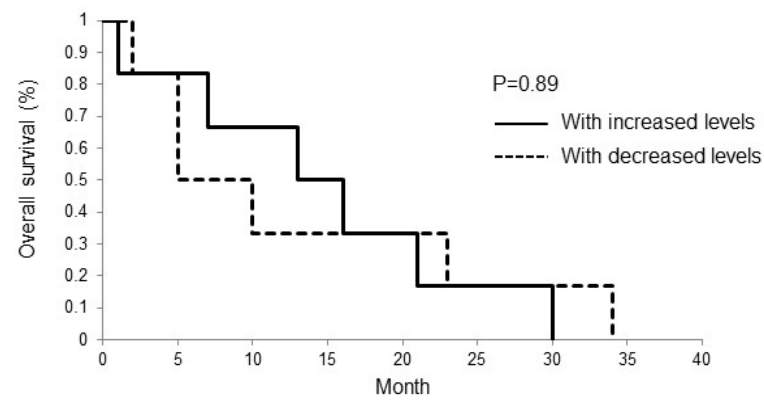

Figure 3. Kaplan-Meier survival curves for overall survival according to (A) relative circ_0024169 levels and (B) the circ 0024169/CUL5 ratio in 12 angiosarcoma patients. Solid and dotted lines represent patients with increased levels and those with decreased levels, respectively.

lower in vascular tumors including pyogenic granuloma and angiosarcoma than in melanoma and SCC (Figure 2B). A significant difference was observed among these groups by the Kruskal-Wallis test $(p=0.0022)$. There were significant changes in the ratio between pyogenic granuloma and melanoma ( $p=0.014)$, SCC $(p=0.025)$, or normal skin $(p=0.0045)$ by Mann-Whitney test. Also, the ratio of angiosarcoma was significantly decreased compared to that of melanoma ( $p=0.040$ ) or normal skin $(p=0.0068)$ : The ratio was lower in pyogenic granuloma compared to angiosarcoma.

Taken together, the result indicated circ_0024169/ CUL5 ratio tended to be reduced in vascular tumors, and seems to be more sensitive than relative levels to distinguish vascular tumors from others.

\subsection{Correlations of circ_0024169 expression and clinical manifestation in patients with angiosarcoma}

Lastly, we tried to determine the clinical significance of relative circ_0024169 levels or circ_0024169/CUL5 ratio in angiosarcoma patients. We found that relative circ_0024169 levels showed mild inverse correlation with the follow-up periods (duration between the first hospital visit and the last hospital visit/the date of death) $(\mathrm{R}=0.28$, Figure $2 \mathrm{C})$. On the other hand, there was no correlation between the ratio and follow-up periods ( $\mathrm{R}$ $=-0.09$, Figure 2D). Accordingly, relative circ 0024169 levels in angiosarcoma tissues may be more useful to predict prognosis than circ_0024169/CUL5 ratio. In Kaplan-Meier method of twelve patients divided into two groups (six patients with increased and decreased relative circ 0024169 levels or circ $0024169 /$ CUL5 ratio), those with decreased relative circ_0024169 levels tended to show poorer prognosis (Figure 3A and 3B), but there was not statistically significant difference.

\section{Discussion}

In this study, we have presented two major findings. First, we found that both relative circ_0024169 levels and circ_0024169/CUL5 ratio was decreased in HDMEC and angiosarcoma cell line in vitro, especially in HDMEC.

On the other hand, circ 0024169/CUL5 ratio was significantly reduced in angiosarcoma and pyogenic granuloma in vivo, which were more evident than relative circ_0024169 levels. A remarkably decreased circ_0024169/CUL5 ratio in HDMEC in vitro may correspond to the reduced levels in pyogenic granuloma in vivo, because pyogenic granuloma is a benign and reactive vascular tumor of the skin. However, circ_0024169 levels showed a mild correlation with the follow-up period. Although there were not significant difference $(p=0.37)$, this might be because of small number of cases: Although circRNA experiments required a mass of RNA, we could not collect enough number of large samples to obtain adequate RNA. Larger study with increased number of patients will be needed in the future. Multiple in vitro experiments using various cell types including normal human keratinocytes, $\mathrm{HaCaT}$ cells, and human umbilical vein endothelial cells (HUVEC) should also be performed. There was no correlation between circ_0024169 / CUL5 ratio and follow-up periods.

Taken together, our pilot study with small patient number suggest the possibility that circ_0024169/CUL5 ratio are useful as a diagnostic biomarker for vascular tumors, whereas relative circ_0024169 levels may have more potential as a prognostic marker of angiosarcoma. Generally, both relative circRNA levels and circRNA levels/linear RNA ratio are important for the evaluation of circRNA expression, and each may have different clinical significance in human diseases: The backsplicing process is thought to be regulated by the ratio of circular to linear transcripts, and the relative abundance of differentially spliced circular isoforms is cell-type specific $(17,18)$. For example, circRNA/linear RNA ratio negatively correlated with the cell proliferation, regardless of whether the linear RNA or circRNA alone was lower or higher expressed in tumor compared to normal tissue (13). However, there have been no studies showing their importance in skin tumors.

In this study, we focused on circ_0024169, 
which were reported to be down-regulated in ovarian cancer and colon cancer (13). As described above, circRNAs are usually resistant to RNase treatment, and more stable than linear RNAs. Because the stable circRNAs tend to accumulate in non-proliferating cells while they are evenly distributed to daughter cells in proliferative cells, the circRNA levels are thought to become relatively low in tumor tissues (13). On the other hand, function of circRNAs in these cancers and angiosarcoma is still unknown. One of the important functions of circRNAs is to bind miRNAs as the sponge and inhibit their functions. miRNAs have already been implicated in the pathogenesis of angiosarcoma: For example, down-regulation of miR-210 stimulates cell proliferation via the induction of E2F3 and EphrinA3 in angiosarcoma (19). The expression of miR-214 and miR-126 are markedly elevated in the plasma of angiosarcoma patients (20). The identification of circ_0024169-assciated miRNAs in angiosarcoma may resulted in the clarification of detailed mechanism of oncogenesis in angiosarcoma.

\section{Acknowledgements}

ISO-HAS was kindly provided by Dr. Mikio Masuzawa (Department of Molecular Diagnostics, School of Allied Health Sciences, Kitasato University, Japan).

\section{References}

1. Elder DE. Lever's Histopathology of the Skin, 10th edn. Wolters Kluwer/Lippincott Williams \& Williams, Philadelphia 2009.

2. Vogt T, Brockmeyer N, Kutzner H, Schofer H. Brief S1 guidelines--Cutaneous angiosarcoma and Kaposi sarcoma. J Dtsch Dermatol Ges. 2013; 11 Suppl 3:2-9, 2-10.

3. Shimozono N, Jinnin M, Masuzawa M, Masuzawa M, Wang Z, Hirano A, Tomizawa Y, Etoh-Kira T, Kajihara I, Harada M, Fukushima S, Ihn H. NUP160-SLC43A3 is a novel recurrent fusion oncogene in angiosarcoma. Cancer Res. 2015; 75:4458-4465.

4. Nigro JM, Cho KR, Fearon ER, Kern SE, Ruppert JM, Oliner JD, Kinzler KW, Vogelstein B. Scrambled exons. Cell. 1991; 64:607-613.

5. Capel B, Swain A, Nicolis S, Hacker A, Walter M, Koopman P, Goodfellow P, Lovell-Badge R. Circular transcripts of the testis-determining gene Sry in adult mouse testis. Cell. 1993; 73:1019-1030.

6. Memczak S, Jens M, Elefsinioti A, et al. Circular RNAs are a large class of animal RNAs with regulatory potency. Nature. 2013; 495:333-338.

7. Hansen TB, Jensen TI, Clausen BH, Bramsen JB, Finsen B, Damgaard CK, Kjems J. Natural RNA circles function as efficient microRNA sponges. Nature. 2013; 495:384-388.

8. Zheng Q, Bao C, Guo W, Li S, Chen J, Chen B, Luo Y, Lyu D, Li Y, Shi G, Liang L, Gu J, He X, Huang S. Circular RNA profiling reveals an abundant circHIPK3 that regulates cell growth by sponging multiple miRNAs. Nat Commun. 2016; 7:11215.

9. Memczak S, Papavasileiou P, Peters O, Rajewsky N. Identification and characterization of circular RNAs as a new class of putative biomarkers in human blood. PloS One. 2015; 10:e0141214.

10. Chen J, Li Y, Zheng Q, et al. Circular RNA profile identifies circPVT1 as a proliferative factor and prognostic marker in gastric cancer. Cancer Lett. 2017; 388:208-219.

11. Galasso M, Costantino G, Pasquali L, Minotti L, Baldassari F, Corra F, Agnoletto C, Volinia S. Profiling of the predicted circular RNAs in ductal in situ and invasive breast cancer: A pilot study. Int J Genomics. 2016; 2016:4503840.

12. Su H, Lin F, Deng X, Shen L, Fang Y, Fei Z, Zhao L, Zhang X, Pan H, Xie D, Jin X, Xie C. Profiling and bioinformatics analyses reveal differential circular RNA expression in radioresistant esophageal cancer cells. J Transl Med. 2016; 14:225.

13. Bachmayr-Heyda A, Reiner AT, Auer K, Sukhbaatar N, Aust S, Bachleitner-Hofmann T, Mesteri I, Grunt TW, Zeillinger R, Pils D. Correlation of circular RNA abundance with proliferation--exemplified with colorectal and ovarian cancer, idiopathic lung fibrosis, and normal human tissues. Sci Rep. 2015; 5:8057.

14. Kanemaru H, Fukushima S, Yamashita J, Honda N, Oyama R, Kakimoto A, Masuguchi S, Ishihara T, Inoue Y, Jinnin M, Ihn H. The circulating microRNA-221 level in patients with malignant melanoma as a new tumor marker. J Dermatol Sci. 2011; 61:187-193.

15. Masuzawa M, Fujimura T, Hamada Y, Fujita Y, Hara H, Nishiyama S, Katsuoka K, Tamauchi H, Sakurai Y. Establishment of a human hemangiosarcoma cell line (ISO-HAS). Int J Cancer. 1999; 81:305-308.

16. Harada M, Jinnin M, Wang Z, Hirano A, Tomizawa Y, Kira T, Igata T, Masuguchi S, Fukushima S, Ihn H. The expression of miR-124 increases in aged skin to cause cell senescence and it decreases in squamous cell carcinoma. Biosci Trends. 2017; 10:454-459.

17. Wang PL, Bao Y, Yee MC, Barrett SP, Hogan GJ, Olsen MN, Dinneny JR, Brown PO, Salzman J. Circular RNA is expressed across the eukaryotic tree of life. PloS One. 2014; 9:e90859.

18. Salzman J, Chen RE, Olsen MN, Wang PL, Brown PO. Cell-type specific features of circular RNA expression. PLoS Genet. 2013; 9:e1003777.

19. Nakashima S, Jinnin M, Kanemaru H, Kajihara I, Igata T, Okamoto S, Tazaki Y, Harada M, Masuguchi S, Fukushima S, Masuzawa M, Amoh Y, Masuzawa M, Ihn H. The role of miR-210, E2F3 and ephrin A3 in angiosarcoma cell proliferation. Eur J Dermatol. 2017; 27:464-471.

20. Heishima K, Mori T, Ichikawa Y, Sakai H, Kuranaga Y, Nakagawa T, Tanaka Y, Okamura Y, Masuzawa M, Sugito N, Murakami M, Yamada N, Akao Y, Maruo K. MicroRNA-214 and MicroRNA-126 are potential biomarkers for malignant endothelial proliferative diseases. Int J Mol Sci. 2015; 16:25377-25391.

(Received March 24, 2019; Revised May 5, 2019; Accepted May 8, 2019) 